2. To: (Receiving organization)

TWR/ENG

3. From: (originating Organization) Consequence Analysis $8 \mathrm{M} 400$

5. Proj./Prog./Dept./Div.:

TWRS FSAR

8. Originator Remarks: For Release.
6. Cog. Engr.:

J. C. Van Keuren N2134

4. Related EDT No.:

NA

7. Purchase Order No.:

NA

9. Equip./Component No.:

NA

10. System/Bldg./Facility:

TWRS TANK FARMS

11. Receiver Remarks:

12. Major Assm. Dug. Mo.:

NA

13. Permit/Permit Application No.: NA

14. Required Response Date: June 14,1996

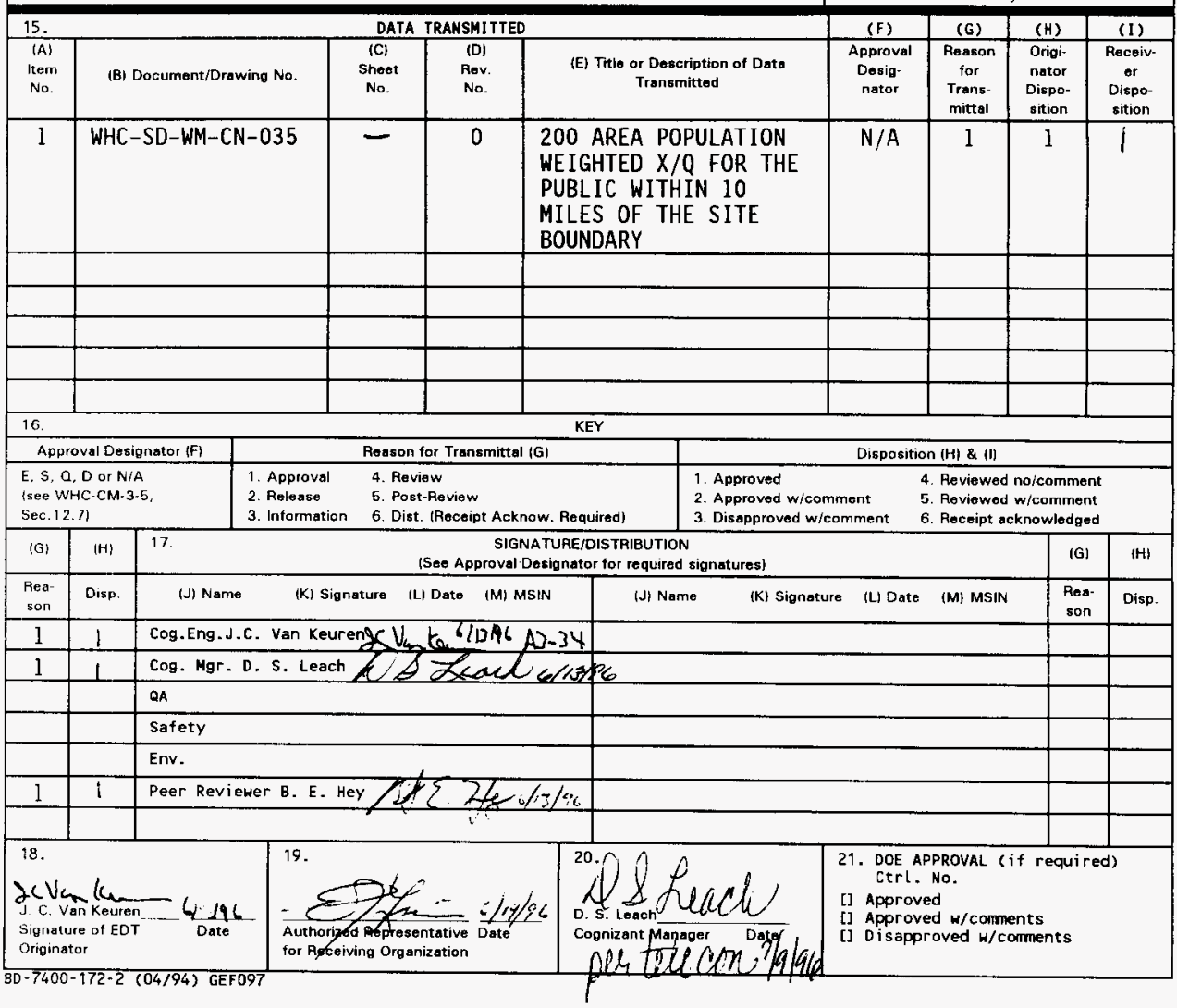




\section{AREA POPULATION WEIGHTED X/Q FOR THE PUBLIC WITHIN 10 MILES OF THE SITE BOUNDARY}

J. C. Van Keuren

Westinghouse Hanford Co, Richland, WA 99352

U.S. Department of Energy Contract DE-AC06-87RL10930

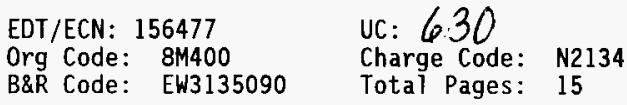

Key Words: $\mathrm{X} / \mathrm{Q}$, Tank Farms, Population

Abstract: An overall population weighted atmospheric dispersion coefficient $(X / Q)$ has been calculated for the public within 10 miles of the Hanford Site boundary. The Columbia river was assumed as the Hanford site boundary to the north and the east. The GXQ code was used for the calculation. The value calculated is $1.88 \times 10^{-8} \mathrm{~s} / \mathrm{m}^{3}$.

IRADEMARK DISCLAIMER. Reference herein to any specific comercial product, process, or service by trade name, trademark, manufacturer, or otherwise, does not necessarily constitute or imply its endorsement, recomendation, or favoring by the United States Goverment or any agency thereof or its contractors or subcontractors.

Printed in the United States of America. To obtain copies of this document, contact: WHC/BCS Document Control Services, P.O. Box 1970, Mailstop H6-08, Richland WA 99352, Phone (509) 372-2420; Fax (509) 376-4989.
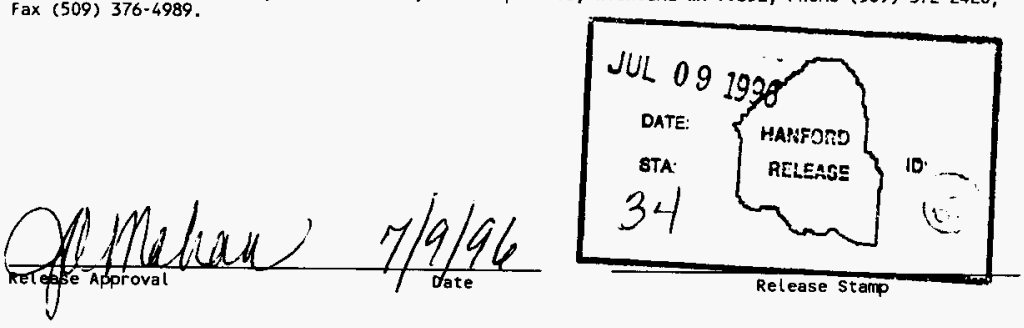

\section{Approved for Public Release}




\section{WHC-SD-WM-CN-035 Rev O}

A calculation of the population weighted atmospheric dispersion coefficients $(X / Q S)$ based on the public located within a 10 mile radius of the Hanford site boundary was performed. The values will be used for comparison with the DOE risk guidelines. The calculation was performed with the GXQ code (Hey 1993) with the following assumptions:

1. The release point was assumed to be the 200 area tank farms. The minimum distances to the site boundary were based on the calculations for the TWRS FSAR (SARR-016). Ten miles $(16,090 \mathrm{~m})$ was added to the distance to determine an area in which population is located. The Columbia river was used as the site boundary to the north and the east.

2. A $X / Q$ averaged over all 16 sector was calculated. The $X / Q$ is population weighted based on population distribution data for the population within $16.1 \mathrm{~km}$ ( $10 \mathrm{miles}$ ) of the site boundary. The $X / Q$ reported is for an average individual within this area. Sector averaging was used to determine a $X / Q$ based on the population being spread across the sector.

3. The $X / Q$ is based on annual average meteorology. Population data are based on 1990 census. Meteorology conditions are calculated based on joint frequency data from 1983 to 1990.

The distances to the site boundary, and the distance plus 10 miles, and the population in each sector are shown in Table 1. The population weighted peak $X / Q$ for the average individual averaged over all 16 sectors is 1.88 E-08. The GXQ input and output files are attached.

\section{References}

Hey, B. E., 1993, GXQ Program Users' Guide, WHC-SD-GN-SWD-30002, Rev. 0, Westinghouse Hanford Company, Richland, Washington.

SARR-016, 1996, Tank Waste Compositions and Atmospheric Dispersions Coefficients for Use in Safety Analysis Consequence Assessments, WHC-SD-WM-SARR-016, Rev. 2, Draft, Westinghouse Hanford Company, Richland, Washington. 
WHC-SD-WM-CN-035 Rev 0

Table 1 Distances and Populations

\begin{tabular}{||l|r|r|r||}
\hline $\begin{array}{c}\text { TRANSPORT } \\
\text { DIRECTION }\end{array}$ & $\begin{array}{c}\text { DISTANCE TO } \\
\text { SITE } \\
\text { BOUNDARY } \\
\text { (m) }\end{array}$ & $\begin{array}{l}\text { DISTANCE } \\
\text { TO SITE } \\
\text { BOUNDARY } \\
\text { PLUS } 10 \\
\text { MILES (m) }\end{array}$ & $\begin{array}{l}\text { POPULATION } \\
\text { WITHIN 10 } \\
\text { MILES 0F } \\
\text { HANFORD SITE } \\
\text { BOUNDARY }\end{array}$ \\
\hline S & 15360 & 31450 & 2842 \\
\hline SSW & 15640 & 31730 & 713 \\
\hline SW & 13875 & 29965 & 1308 \\
\hline WSW & 11100 & 27190 & 1956 \\
\hline W & 11100 & 27190 & 771 \\
\hline WNW & 11100 & 27190 & 641 \\
\hline NW & 11440 & 27530 & 548 \\
\hline NNW & 8690 & 24780 & 544 \\
\hline N & 8760 & 24850 & 434 \\
\hline NNE & 10610 & 26700 & 268 \\
\hline NE & 10680 & 26770 & 393 \\
\hline ENE & 10530 & 26620 & 423 \\
\hline E & 12630 & 28720 & 452 \\
\hline ESE & 18730 & 34820 & 289 \\
\hline SE & 22440 & 38530 & 1141 \\
\hline SSE & 19960 & 36050 & 2796 \\
\hline
\end{tabular}


WHC-SD-WM-CN-035 Rev 0

Attachment I GXQ Input File 
Population based on 1 mile from river bd annual average meteorology c GXQ Version 4.0 Input File

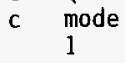

$c$

c MODE CHOICE:

$c$ mode $=1$ then $X / Q$ based on Hanford site specific meteorology

$c$ mode $=2$ then $X / Q$ based on atmospheric stability class and wind speed

$c$ mode $=3$ then $X / Q$ plot file is created

C

c LOGICAL CHOICES:

c ifox inorm icdf ichk isite ipop

$c i f o x=t$ then joint frequency used to compute frequency to exceed $x / Q$

$c=f$ then joint frequency used to compute annual average $x / 0$

$c$ inorm $=t$ then joint frequency data is normalized (as in GENII)

$c \quad=f$ then joint frequency data is un-normalized

$c$ icdf $=t$ then cumulative distribution file created (CDF.OUT)

$c \quad=f$ then no cumulative distribution file created

$c$ ichk = $t$ then $X / Q$ parameter print option turned on

$c \quad=f$ then no parameter print

$c$ isite $=t$ then $X / Q$ based on joint frequency data for all 16 sectors

$c=f$ then $X / Q$ based on joint frequency data of individual sectors

$c$ ipop $=t$ then $X / Q$ is population weighted

$c=f$ then no population weighting

$\mathrm{c}$

c $X / Q$ AND WIND SPEED ADJUSTMENT MODELS:

$C$ ipuff idep isrc iwind

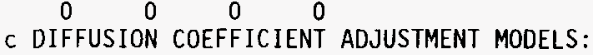

C iwake ipm iflow ientr

c EFFECTIVE RELEASE HEIGHT ADJUSTMENT MODELS:

C (irise igrnd) iwash igrav

$\begin{array}{llll}0 & 0 & 0 & 0\end{array}$

c ipuff $=1$ then $X / Q$ calculated using puff model

$c=0$ then $X / Q$ calculated using default continuous $p l u m e$ mode 1

$c$ idep $=1$ then plume depletion model turned on (Chamberlain model)

$c$ isrc $=1$ then $X / Q$ multiplied by scalar

$c \quad=2$ then $X / Q$ adjusted by wind speed function

$c$ iwind $=1$ then wind speed corrected for plume height

$c$ iwake $=1$ then NRC RG 1.145 building wake model turned on

$\mathrm{c} \quad=2$ then MACCS virtual distance building wake model turned on

c $\mathrm{ipm}=1$ then NRC RG 1.145 plume meander model turned on

$\mathrm{c}=2$ then 5 th Power Law plume meander model turned on

c $=3$ then sector average model turned on

$c$ iflow $=1$ then sigmas adjusted for volume flow rate

$c$ ientr $=1$ then method of Pasquill used to account for entrainment

c irise $=1$ then MACCS buoyant plume rise model turned on

$\mathrm{c}=2$ then ISC2 momentum/buoyancy plume rise model turned on 


\section{WHC-SD-WM-CN-035 Rev 0}

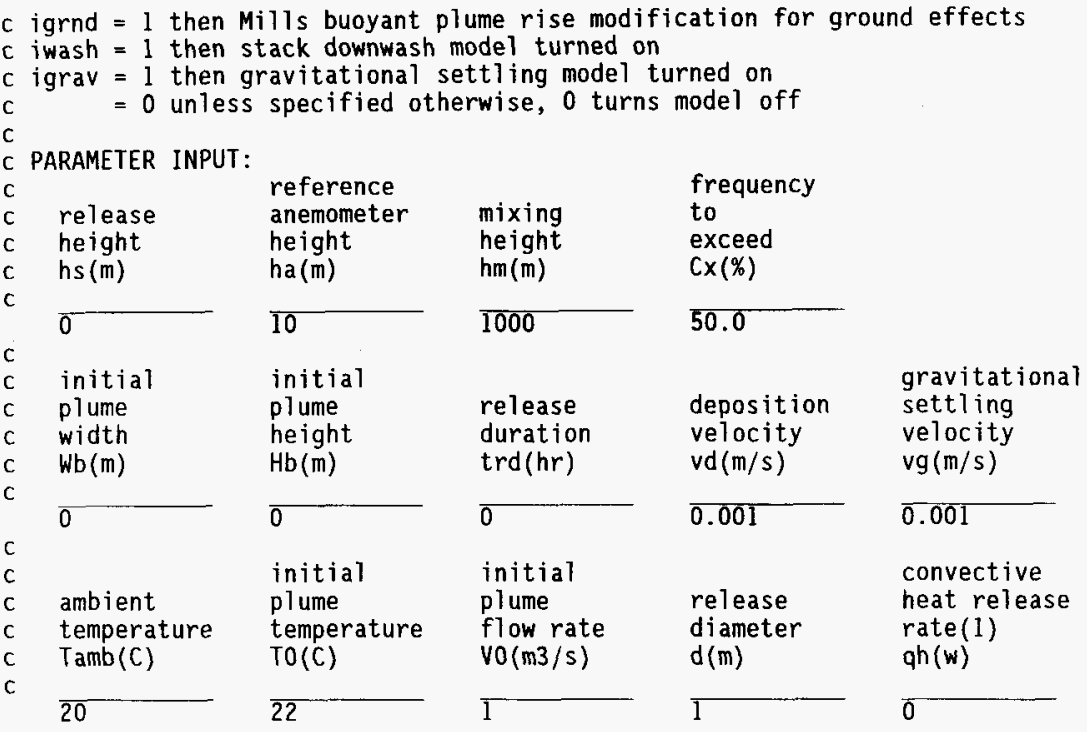

\footnotetext{
(1) If zero then buoyant flux based on plume/ambient temperature difference. 
WHC-SD-WM-CN-035 Rev 0

c imax $=$ distance intervals

c $y \max =$ maximum offset to plot (m)

c jmax $=$ offset intervals

c xamin = minimum scaled $X / Q$ to calculate

c power = exponent in power function step size

$131450 \quad 0$

$231730 \quad 0$

$3 \quad 29965 \quad 0$

$\begin{array}{lll}4 & 27190 & 0\end{array}$

$5 \quad 27190 \quad 0$

$6 \quad 27190 \quad 0$

$\begin{array}{lll}7 & 27530 & 0\end{array}$

$824780 \quad 0$

$924850 \quad 0$

$1026700 \quad 0$

$1126770 \quad 0$

$\begin{array}{lll}12 & 26620 & 0\end{array}$

$13 \quad 28720 \quad 0$

$14 \quad 34820 \quad 0$

$\begin{array}{lll}15 & 38530 & 0\end{array}$

$16 \quad 36050 \quad 0$ 
WHC-SD-WM-CN-035 Rev 0

Attachment 2 GXQ Output 
WHC-SD-WM-CN-035 Rev 0

Current Input File Name: ragpop. IN

GXQ Version 4.0

December 19, 1994

General Purpose Atmospheric Dispersion Code

Produced by Westinghouse Hanford Company

Users Guide documented in WHC-SD-GN-SWD-30002 Rev. 1.

Validation documented in WHC-SD-GN-SWD-30003 Rev. 1.

Code Custodian is: Brit E. Hey

Westinghouse Hanford Company

P.0. Box 1970

Richland, WA 99352

(509) $376-2921$

Run Date $=06 / 12 / 96$

Run Time $=08: 15: 22.87$

INPUT ECHO:

Population based on 1 mile from river bd annual average meteorology

c GXQ Version 4.0 Input File

C mode

1

$C$

c MODE CHOICE:

$c$ mode $=1$ then $X / Q$ based on Hanford site specific meteorology

$c$ mode $=2$ then $X / Q$ based on atmospheric stability $c l a s s$ and wind speed

$c$ mode $=3$ then $X / Q$ plot file is created

c

c LOGICAL CHOICES:

c ifox inorm icdf ichk isite jpop

$c$ ifox $=t$ then joint frequency used to compute frequency to exceed $x / Q$

$c=f$ then joint frequency used to compute annual average $X / Q$

$c$ inorm $=t$ then joint frequency data is normaljzed (as in GENII)

$c \quad=f$ then joint frequency data is un-normalized

$c$ icdf $=t$ then cumulative distribution file created (CDF.OUT)

$c=f$ then no cumulative distribution file created

$c$ ichk $=t$ then $X / Q$ parameter print option turned on

$c \quad=f$ then no parameter print

$c$ isite $=t$ then $X / Q$ based on joint frequency data for all 16 sectors

$c \quad=f$ then $X / Q$ based on joint frequency data of individual sectors

$c$ ipop $=t$ then $X / Q$ is population weighted 


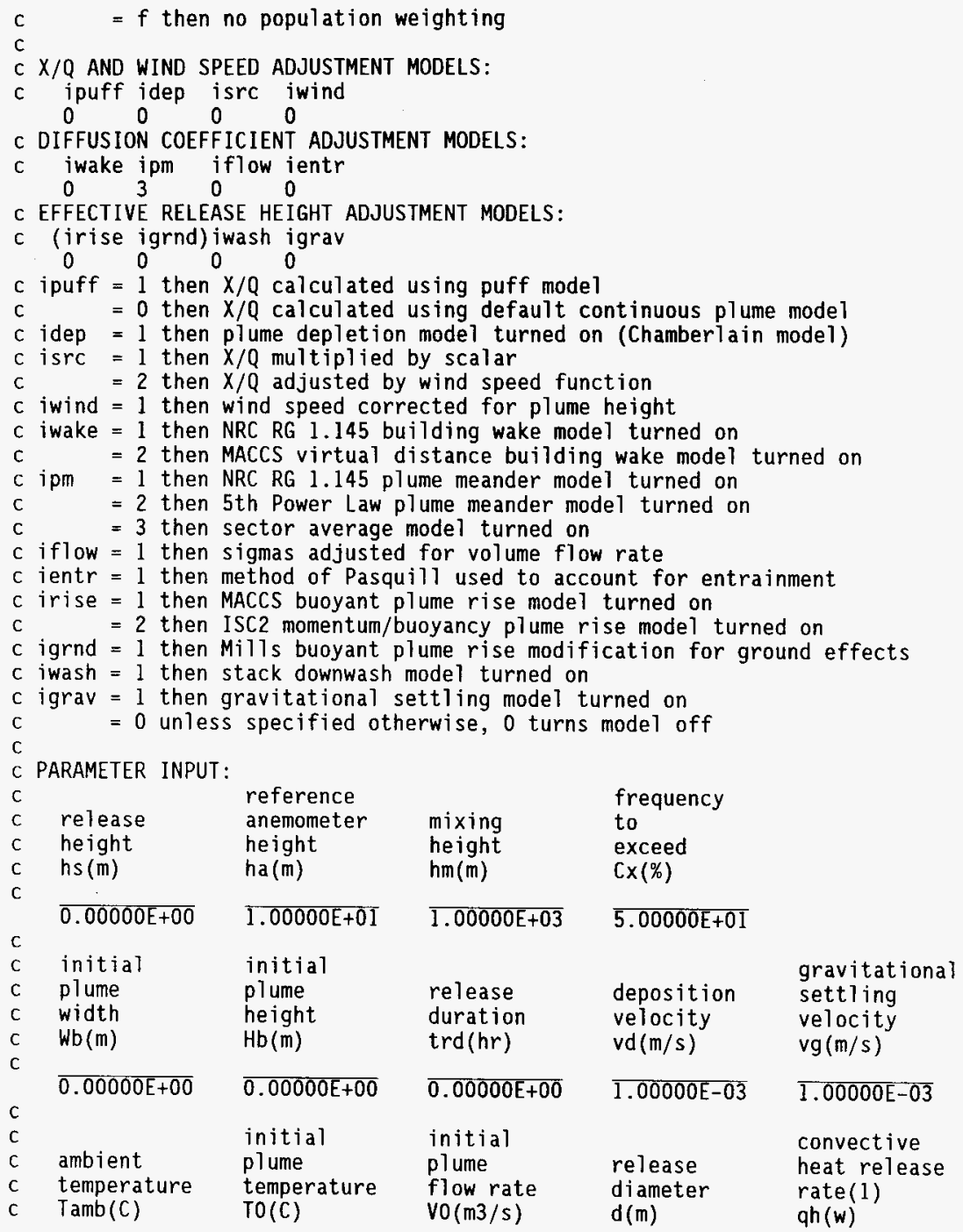


WHC-SD-WM-CN-035 Rev 0

C

$\overline{2.00000 E+01} \quad \overline{2.20000 E+01} \quad \overline{1.00000 E+00} \quad \overline{1.00000 E+00} \quad \overline{0.00000 E+00}$

c (1) If zero then buoyant flux based on plume/ambient temperature difference.

C

c $X / Q \quad$ Wind

c scaling Speed

c factor Exponent

c c(?) a(?)

$\mathrm{c}$

C

$\overline{1.00000 E+00} \quad \overline{7.80000 E-01}$

c RECEPTOR DEPENDENT DATA (no line $1 \mathrm{imit}$ )

C FOR MODE make

RECEPTOR DEPENDENT DATA

c 1 (site specific)

sector distance receptor-height

c 2 (by class \& wind speed) class windspeed distance offset receptor-height

c 3 (create plot file) class windspeed xmax imax ymax jmax xqmin power

C

C RECEPTOR PARAMETER DESCRIPTION

c sector $=0,1,2 \ldots($ all, S, SSW, etc. $)$

c distance $=$ receptor distance $(\mathrm{m})$

c receptor height $=$ height of receptor $(\mathrm{m})$

c class $=1,2,3,4,5,6,7$ (P-G stability class $A, B, C, D, E, F, G)$

c windspeed $=$ anemometer wind speed $(\mathrm{m} / \mathrm{s})$

c offset = offset from plume centerline $(\mathrm{m})$

$c \max =$ maximum distance to plot or calculate to (m)

c $i \max =$ distance intervals

c $y \max =$ maximum offset to plot (m)

c $j \max =$ offset intervals

c $x$ gmin $=$ minimum scaled $X / Q$ to calculate

$c$ power = exponent in power function step size

MODE :

Site specific $X / Q$ calculated.

LOGICAL CHOICES:

Joint frequency used to calculate annual average $X / Q$.

No normalization of joint frequency.

$X / Q$ calculated for overall site.

$X / Q$ is population weighted.

MODELS SELECTED:

Sector average model selected.

Default Gaussian plume model selected.

WARNING/ERROR MESSAGES:

JOINT FREQUENCY DATA:

200 AREA (HMS) - 10 M - Pasquil1 A - G (1983 - 1991 Average)

Created $8 / 26 / 92$ KR 


\section{WHC-SD-WM-CN-035 Rev 0}

POPULATION DATA:

Population within $80 \mathrm{~km}$ of the Hanford 200 Area, 1990 Census (Beck, et al 1991) Created 10/21/91 KR, Revised 2/4/92 KR

Population based on 1 mile from river bd annual average meteorology

\begin{tabular}{|c|c|c|c|c|c|c|c|c|}
\hline ECTOR & $\begin{array}{l}\text { DISTANCE } \\
(\mathrm{m})\end{array}$ & $\begin{array}{c}\text { RECEPT } \\
\text { HEIGHT } \\
\text { (m) }\end{array}$ & $\begin{array}{c}\text { SECT. } \\
\text { FREQ. } \\
(\%)\end{array}$ & POPULATION & $\begin{array}{c}\text { TOTAL } \\
\text { POPULATION } \\
\text { SCALED } \\
X / Q \\
(s / \mathrm{m} 3)\end{array}$ & $\begin{array}{c}\text { AVERAGE } \\
\text { INDIVIDUAL } \\
\text { SCALED } \\
X / Q \\
(\mathrm{~s} / \mathrm{m} 3)\end{array}$ & $\begin{array}{l}\text { ATM. } \\
\text { STAB. } \\
\text { CLASS }\end{array}$ & $\begin{array}{l}\text { WIND } \\
\text { SPEED } \\
\text { (m/s) }\end{array}$ \\
\hline & 36050 & 4 & 99.94 & 15519 & $2.92 \mathrm{E}-04$ & $88 \mathrm{E}-08$ & & \\
\hline
\end{tabular}


WHC-SD-WM-CN-035 Rev 0

Attachment 3 PEER/HEDOP REVIEW CHECKLISTS 


\section{WHC-SD-WM-CN-035 Rev 0}

\section{PEER REVIEW CHECKLIST}

Document Reviewed: 200 Area Population Weighted $X / Q$ for the Public Within 10 Miles of the Site Boundary, WHC-SD-WM-CN-035

Author: J. C. Van Keuren

Date: 6/14/96

Scope of Review: Entire Document
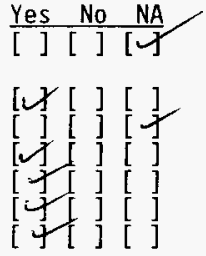

[ ] [ ] H

[于[ ] [ ]

[ ] [ ] [ [ U

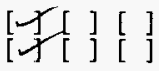

[ ] [ ] [ $[X$

[ ] ] [ ] ] [ [ [ [

$[\mathcal{X}[\mathrm{]}] \mathrm{C}$

[ ] [ ] ] [

[ ] [ ] [ [ [ i

[4] [ [ ]
Previous reviews complete and cover analysis, up to scope of this review, with no gaps.

Problem completely defined.

Accident scenarios developed in a clear and logical manner.

Necessary assumptions explicitly stated and supported.

Computer codes and data files documented.

Data used in calculations explicitly stated in document.

Data checked for consistency with original source information as applicable.

Mathematical derivations checked including dimensional consistency of results.

Models appropriate and used within range of validity or use outside range of established validity justified.

Hand calculations checked for errors. Spreadsheet results should be treated exactly the same as hand calculations.

Software input correct and consistent with document reviewed.

Software output consistent with input and with results reported in document reviewed.

Limits/criteria/guidelines applied to analysis results are appropriate and referenced. Limits/criteria/guidelines checked against references.

Safety margins consistent with good engineering practices.

Conclusions consistent with analytical results and applicable

limits.

Results and conclusions address all points required in the problem statement.

Format consistent with appropriate NRC Regulatory Guide or other standards

Review calculations, comments, and/or notes are attached.

Document approved.
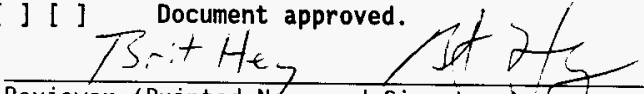

Reviewer (Printed Name and Signature)

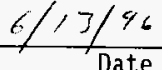




\section{WHC-SD-WM-CN-035 Rev 0}

\section{HEDOP REVIEW CHECKLIST}

Document Reviewed: $\quad 200$ Area Population Weighted $x / Q$ for the Public Within 10 Miles of the Site Boundary, WHC-SD-WM-CN-035

Author: $\quad$ J. C. Van Keuren

Date: $\quad 6 / 14 / 96$

Scope of Review: $\quad$ Entire Document

YES NO* N/A

[ ] [ ] [Y 1. A detailed technical review and approval of the environmenta] transport and dose calculation portion of the analysis has

[4 [ ] [ ] 2. Detailed technical review(s) and approval(s) of scenario and release determinations have been performed and documented.

[ ] [ ] [ 3 . HEDOP-approved code (s) were used.

Receptor locations were selected according to HEDOP recommendations.

[ ] [ ] [ 5. All applicable environmental pathways and code options were

[ [ ] [ ] 6. Hanford site data were used. included and are appropriate for the calculations.

[ ] [ ] [ 7. Mode] adjustments external to the computer program were

[] [ ] justified and performed correctly.

[] [ ] [ \&. The analysis is consistent with HEDOP recommendations. Supporting notes, calculations, comments, comment resolutions, or other information is attached. (Use the "Page 1 of $X "$ page numbering format and sign and date each added page.)

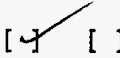

10. Approval is granted on behalf of the Hanford Environmental Dose Overview Panel.

* A11 "NO" responses must be explained and use of nonstandard methods justified.

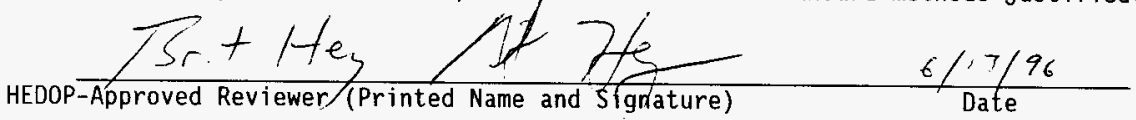

COMMENTS (add additional signed and dated pages if necessary): 


\section{DISTRIBUTION SHEET}

\begin{tabular}{|c|c|c|c|c|c|}
\hline \multirow{2}{*}{$\begin{array}{l}\text { To } \\
\text { Distribution }\end{array}$} & \multirow{2}{*}{\multicolumn{3}{|c|}{$\begin{array}{l}\text { From } \\
\text { Consequence Analys is }\end{array}$}} & \multicolumn{2}{|l|}{ Page 1 of 1} \\
\hline & & & & \multicolumn{2}{|c|}{ Date July 8, 1996} \\
\hline \multicolumn{4}{|l|}{ Project Title/Work Order } & \multicolumn{2}{|c|}{ EDT No. 156477} \\
\hline \multicolumn{4}{|c|}{$\begin{array}{l}200 \text { Area Population Weighted } X / Q \text { for the Public Within } 10 \text { Miles } \\
\text { of the Site Boundary }\end{array}$} & \multicolumn{2}{|l|}{ ECN No. } \\
\hline Name & MSIN & $\begin{array}{c}\text { Text } \\
\text { With All } \\
\text { Attach. }\end{array}$ & Text Only & $\begin{array}{l}\text { Attach./ } \\
\text { Appendix } \\
\text { Only }\end{array}$ & $\begin{array}{l}\text { EDT/ECN } \\
\text { Only }\end{array}$ \\
\hline
\end{tabular}
B. E. Hey
A3-34 $X$
D. S. Leach
A3-34 $X$
E. J. Lipke
A2-34
J. C. Van Keuren
A3-34
Central Files 